\title{
Using handheld devices for real-time wireless Teleconsultation
}

\author{
K. A. Banitsas ${ }^{1}$, P. Georgiadis ${ }^{2}$, S. Tachakra ${ }^{3}$, D. Cavouras ${ }^{2}$ \\ ${ }^{1}$ Department of Electrical and Computer Engineering, Brunel University, West London, UK \\ ${ }^{2}$ Medical Image and Signal Processing Lab, Technological Educational Institution of Athens, Egaleo, Athens, Greece. \\ ${ }^{3}$ A\&E Department, North West London Hospitals NHS Trust, Central Middlesex Hospital, London, UK
}

\begin{abstract}
Recent advances in the hardware of handheld devices, opened up the way for newer applications in the healthcare sector, and more specifically, in the Teleconsultation field. Out of these devices, this paper focuses on the services that Personal Digital Assistants and smartphones can provide to improve the speed, quality and ease of delivering a medical opinion from a distance and laying the ground for an allwireless hospital. In that manner, PDAs were used to wirelessly support the viewing of DICOM images and to allow for mobile videoconferencing while within the hospital. Smartphones were also used to carry still images, multiframes and live video outside the hospital. Both of these applications aimed at increasing the mobility of the consultant while improving the healthcare service.
\end{abstract}

Keywords - teleconsultation, PDA, wireless, mobile

\section{INTRODUCTION}

The increased use of mobile devices, Personal Digital Assistants (PDAs) and wireless devices in general, have changed the way we perceive things around us and have had a definite impact on our working environment. These kinds of changes also had a great effect on the healthcare sector, where the need for accurate and fast delivery of information is critical.

Currently, ongoing research projects like MedLAN [1], [2] opened up the way for wireless transfer of real-time video, audio and information. In particular, the MedLAN system is dedicated for use in the Accidents and Emergency (A\&E) ward of the hospitals and allow the treating doctor to communicate (using videoconferencing) with a consultant that may reside either within or outside the hospital through the use of a wireless link. The whole trolley is very light and there are even versions that can be wearable.

A prototype of the system has been tested for the last three years in the North West London Hospitals and received very positive remarks from both the treating doctors and the consultants, as it allows for easier, more reliable, faster and cheaper Teleconsultation [3].

Despite the autonomy that such a system gives to the personnel operating inside the A\&E department, there is still the obvious need for mobility in the consultant's site. Consultants are rarely in a fixed position, as they have to move from one ward of the hospital to another. Furthermore, there should exist a way of getting in touch with the patient's information while being away from the hospital and effectively help on delivering a diagnosis as accurate as possible.
The former need can be satisfied with the use of portable Personal Digital Assistants and wireless LANs, while the latter can be achieved by using mobile phones capable of carrying out sophisticated tasks (smartphones). The objective of this paper is to suggest an all-wireless environment for Teleconsultation: from the A\&E ward of the hospital (using mobile computers and WLANs) to the consultant (using PDAs, WLANs and smartphones), all healthcare personnel should be allowed freedom of movement.

\section{Current Work}

Today, as PDA's technical characteristics improve, an increasingly large number of new applications emerge. With hardware specifications that can compete with an average desktop computer (CPUs running at $400 \mathrm{MHz}$, flash memory of $256 \mathrm{MB}$, TFT colour screens, internal support for wireless network and even fingerprint security), PDAs found their way into a large number of professional applications, including the healthcare delivery.

However, within the healthcare sector, so far they have been limited into providing one of the following [4], [5], [6], [7]:

- Support for patient database access for drug prescription: healthcare staff found PDAs a convenient tool for bed-to-bed survey and update of patient information and drug prescription.

- Wired or wireless view of medical imaging: Despite their screen size limitation, PDAs can still display efficiently a still medical image (x-rays, MRI, CT, etc). Zooming in and measurement abilities provide an extra tool for doctors or consultants while allowing for complete mobility.

- Support for general applications: These include appointment keeping, Internet access, network support, printing, etc.

For the Telemedical application to exist outside the hospital boundaries, there is the need for a communication path. Some projects exist around the world that offer WLAN support in hotspots around a city and even envision a WLAN coverage that would include entire cities [8]. However, in reality, these kinds of applications usually involve some kind of connection through a mobile phone.

The third generation mobile telephony $(3 \mathrm{G})$ will play an important role into that direction. However, $3 \mathrm{G}$ is not 
available in most of the countries worldwide and even if it is, it is currently limited in covering urban regions.

Second generation mobile telephony $(2 \mathrm{G})$, has long been established around the world. With its addition of General Packet Radio Service (GPRS), it can support basic data transfer of typically $40 \mathrm{Kbps}(2.5 \mathrm{G})$ and can assist the transfer of medical information to either a mobile phone, or a computer connected to that mobile phone, at a modest speed.

Newer mobile phone devices take advantage of the data abilities offered by the $2.5 \mathrm{G}$. That, coupled with their increasing computational abilities, can allow the consultant to effectively use these devices in the place of PDAs, while outside the hospital.

In conclusion, PDAs have been proven to perform tasks beyond those that are used for today, while mobile phones act more and more like PDAs. For the remainder of this paper, there will be a research into the notion that PDAs can be used for live wireless videoconferencing while within WLAN support (inside the hospital), while smartphones can be used for direct access to medical imaging / video (outside the hospital).

\section{METHODOLOGY}

For the case of videoconferencing using a PDA, the following procedure was adopted:

The hardware initially chosen was the Compaq iPaq 3630 combined with D-Link's IEEE 802.11b compliant wireless card (DCF-660W). The device features a $200 \mathrm{MHz}$ Intel Strong-Arm Processor, 32 MB SDRAM, 16 MB Flash ROM, a CF Card expansion slot for adding additional memory capabilities and a 3.8" 240x320 16-bit color TFT display. The operating system is the Microsoft Pocket PC 2002. Later on, the entire PDA device was updated using the iPAQ 5500 that supported WLAN internally (IEEE $802.11 \mathrm{~b}$ ) having a top transfer speed of $11 \mathrm{Mbps}$ and a practical speed of about $3 \mathrm{Mbps}$.

A number of Access Points (AP) was laid around the Central Middlesex Hospital (CMH) and were connected to the wired backbone of the hospital and allowed for wireless connection between the PDA and the hospital network. They also allowed for roaming from one access point to another while being seamlessly connected to the network. This gave the consultant the opportunity of using the PDA for any kind of network application, anywhere in the hospital, as long as there will be WLAN coverage. The same scenarios were repeated in Northwick Park London Hospital and Wembley Hospital and produced similar results.

A simple videoconferencing server (Windows Media encoder 9) was used, able to broadcast a videostream at a specific IP address. Within the PDA, Windows Media Player was used to connect to that specific IP and display the videostream transmitted from the $A \& E$ ward to the consultant The overall procedure was quite simple and only required entering an IP number to the video client. Since the hospital was using static IPs this information need only to be entered once.

For static image viewing, a simple DICOM viewer was built, even though there were commercially available DICOM viewers to be used with PDAs. In addition to simple image viewing, they supported several functions like zooming into the Region Of Interest (ROI), measuring tools and performing basic filtering (equalize, invert, median, masking, etc). Apart from viewing DICOM format, the PDA could also support a wide range of standard formats (BMP, JPEG, GIF, PNG, PCX, TIFF).

For the case of transferring medical information directly into a smartphone, the following steps took place:

An appropriate mobile device was chosen from a wide range of alternatives. The new Nokia 6600 seemed like a solid candidate as it supported the new Symbian 60 series operating system. The screen size was $208 \times 176$ pixels displayed in a $65 \mathrm{k}$ colours TFT screen. It had internal support for Internet browsing, email and viewing streaming media. $128 \mathrm{MB}$ flash memory was added to support storage of large videos and images. The phone had internal support for viewing standard graphic formats, but not DICOM. For that reason, a simple DICOM viewer was built using the Series 6 SDK for Symbian OS, capable of performing some basic image manipulations.

The link between the mobile phone device and the network was chosen to be GPRS instead of HSCSD as packet radio was proven much more convenient.

Finally, it is worth mentioning that a number of smartphone devices have appeared on the market today running a compatible operating system and having even better technical specifications (Sony-Ericsson P900, etc). Furthermore, devices like the new Motorola MPx200 smartphone series embed the newer Windows Mobile 2003 operating system that allowed for an even better software development.

\section{RESULTS}

The PDA performed as expected, while viewing static images. The screen size, although smaller than a conventional computer, seemed to be adequate (especially with the use of zooming tools) [Fig. 1]. The wireless network support allowed for more than $3 \mathrm{Mbps}$ bandwidth, which was proven more than enough for image and video transfer. The DICOM images could be saved into either locally or network drive, printed or used to amend a patient's record [9]

On the videoconferencing part, the image quality was sufficient and could even display accurately ECG traces or ultrasound monitors [Fig. 1]. There was, however, a delay of 
5-9 seconds between the sender and the receiver that was mainly attributed to the frames that had to be buffered and compressed by the sender, before transmitting the videostream. Since the compression algorithm for the videostream was related to H.263 (a subset of the MPEG 4 family), it was clear that the video quality much depended on the speed that the camera (or the objects) were moving: the faster the speed, the lower the quality.

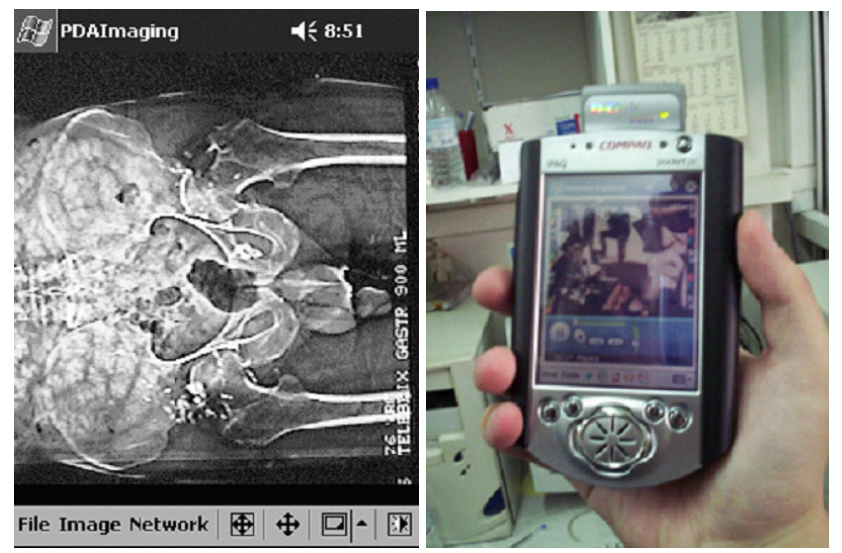

Fig. 1. Displaying a DICOM image on the PDA screen (left), live wireless videoconferencing using a PDA (right)

The smartphone application produced three different sets of results:

- High resolution still images: These images were usually transferred through GPRS as a Multimedia Message Service (MMS) either at a standard JPEG format with a modest compression, or as native DICOMs. JPEGs could be viewed directly into the smartphone's screen with various levels of zooming in/out. DICOM images used a self-developed application that allowed for some extra features for image manipulation [Fig. 2]. A single MMS could handle up to $100 \mathrm{~KB}$ of data; but this value heavily depended on the mobile service provider. Sending a larger image is feasible, but at higher cost, as it had to be broken down in blocks of $100 \mathrm{~KB}$.

- Multi-frame DICOM images: Some modalities require the use of multiple frames stored in a single DICOM file. These files can be transmitted to the smartphone either as DICOM (to be viewed using a special programme), or converted to a " $3 \mathrm{gp}$ " format that can be accepted by most of the smartphones today. The latter can be viewed in the media player that is embedded in the smartphone. Tools like zooming in/out and adjusting the delay between frames, are also available [Fig. 2].

- Live video broadcast: Modern smartphones have internal support for streaming audio and video. By setting up a broadcast server in the hospital's site, the mobile phone can connect to a specific site and view the live videostream transmitted by the hospital. The video window was $176 \times 144$ with a frame rate of 5-10 fps. Although the screen resolution of the smartphone is relatively low, there are a number of telemedical applications that would benefit by it, including viewing an ECG traces, direct view of a patient, etc.

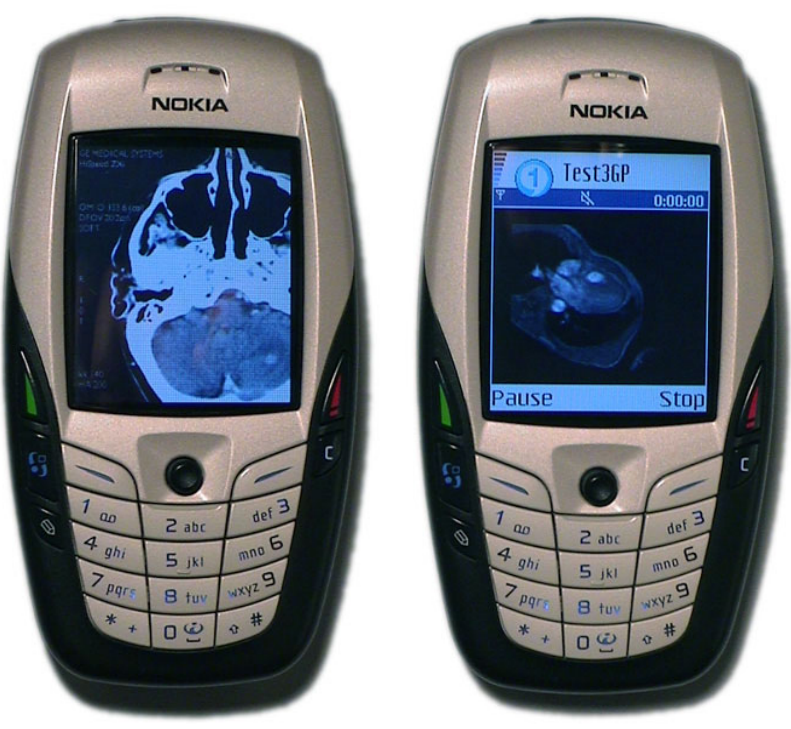

Fig. 2. Using a smartphone to: view a still DICOM image (left), view a multiframe DICOM image(right)

\section{DISCUSSION}

The above experiments are part of an ongoing research project with a lot of room for improvement. Fortunately, frequent advances in the hardware of handheld devices, will serve as a stepping-stone for these kinds of improvements.

Nevertheless, both applications seemed to perform very well considering the tasks initially set.

Some problems with the video delay while videoconferencing using a PDA, can be improved by using faster processors and WLANs supporting higher bandwidth (IEEE $802.11 \mathrm{~g}$ ). The replacement of IEEE 802.11b APs with IEEE 802.11a was proven inadequate, as despite the higher bandwidth, the reduced active range of the "a" technology dictated that at least four times as many "a" APs had to be used, to cover the same space [10]

For the smartphone, the best application was proven to be the transfer of still DICOM images to be viewed on the smartphone's screen. Video seemed to be of a relatively low quality and the frame rate was reduced, as it was related to the data speed offered by the mobile provider (maximum of 40Kbps).

Finally, the security of the system was high enough to justify transferring medical data:

On the PDA, several security measures were implemented including Wired Equivalent Privacy (WEP), SSID, fingerprint scan, username / password access or even encapsulation into an IPSec protocol [11]. 
The smartphone applications seemed to be adequately secure by relying in the internal security of the GSM system.

Overall, both applications were tested at their prototype state by several consultants and received a number of positive remarks, mainly related to the substantial increase of mobility that these offer to both doctors and consultants [5].

\section{CONCLUSIONS}

As our technological society is moving towards an allwireless healthcare, the importance of projects using small and portable mobile devices to effectively assist healthcare personnel becomes increasingly apparent.

Using powerful PDAs equipped with WLAN support and mobile phones with advanced operating system, capable of running sophisticated software, can assist the job of the consultant either by videoconferencing from anywhere within the hospital, or having access to patient information and imaging, while outside the hospital. This will increase both the mobility of consultants (translating to a reduced time between consultations) and the speed of medical diagnosis by minimising the periods that a consultant will be unavailable.

Finally, some initial hardware related problems can be improved by taking advantage of improvements in mobile technology.

\section{REFERENCES}

[1] R.S.H. Istepanian, Sapal Tachakra, Konstantinos A. Banitsas "Medical Wireless LAN Systems (MedLAN). State of the Art, Challenges, and Future Directions", eHealth conference, City University, April 2001

[2] Konstantinos A. Banitsas, R.S.H. Istepanian, Sapal Tachakra, "Applications of Medical Wireless LAN Systems (MedLAN)", IJMM journal, Sep 2000

[3] Konstantinos A. Banitsas, Sapal Tachakra, Yong Hua Song, "Adjusting DICOM Specifications When Using Wireless LANs: The MedLAN example" to be presented to IEEE EMBC conference, Cancun, Mexico, Sep 2003

[4] Rafael Andrade, et. al., "A strategy for a wireless patient record and image data", International Congress Series, 2003, 1256, pp. 869-872

[5] Schweitzer T., et. al., "Teleradiology on a Personal Digital Assistant", Mocomed workshop 2002

[6] Lynn Easters, "Use of Personal Digital Assistants for point-of-care trauma documentation", Journal of emergency nursing, 27:5, Oct 2001, pp.516-518

[7] U. Engelmann, et. al., "Mobile teleradiology: all images everywhere", International Congress Series, 2001, 1230, pp. 844-850

[8] A. Richard, "Manchester launches first urban WLAN", Netimperative publications, 2001

[9] Steve B. Merritt, John D. Enderle, Jeffrey V. Bronke, "POCKET PACS: A DICOM Viewing Application for the Pocket PC 2002 Operating System", IEEE EMBC 2003, pp. 1376-1377

[10] Konstantinos A. Banitsas, Sapal Tachakra, R.S.H. Istepanian, "Operational Parameters of a Medical Wireless LAN: Security, Range and Interference issues", IEEE EMBC conference, Houston, Oct 2002

[11] Thomas J. Owens, Sapal Tachakra, Konstantinos A. Banitsas, Robert S. H. Istepanian, "Securing a Medical Wireless LAN System", IEEE EMBC conference, Istanbul, Oct 2001. 